\title{
Sirtuin 6 and metabolic genes interplay in Warburg effect in cancers
}

\author{
Nosayba Al-Azzam* \\ Department of Physiology and Biochemistry, Jordan University of Science and Technology, P.O. Box 3030, Irbid 22110, Jordan
}

(Received 14 November, 2019; Accepted 13 December, 2019; Published online 14 February, 2020)

\begin{abstract}
Under oxygen availability, normal cells undergo mitochondrial oxidative phosphorylation to metabolize glucose and yield up to 36 ATPs per glucose molecule for cellular functions, and undergo nonoxidative metabolism (glycolysis) under hypoxic and proliferating conditions to yield 2 ATP per glucose. These cells metabolize glucose to pyruvate via glycolysis followed by conversion of pyruvate to lactate via lactate dehydrogenase. However, cancer cells have the ability to undergo glycolysis and ferment glucose to lactate regardless of oxygen availability; a phenomenon first addressed by Otto Warburg and called, "Warburg effect". Numerous glycolytic genes/proteins have been identified in tumors; that include glucose transporter 1 (GLUT1), hexokinase 2 (HK2), pyruvate kinase-M2 splice isoform (PKM2), and lactate dehydrogenase (LDHA). Histone deacetylase sirtuin 6 (SIRT6), an epigenetic regulator, is highly expressed in various cancers. SIRT6 plays an important role in Warburg effect by regulating many glycolytic genes. Loss of SIRT6 enhances tumor growth via enhancing glycolysis. This review is mainly concerned with exploring the most recent advances in understanding the roles of the metabolic genes (GLUT1, HK2, PKM2, and LDH-A) and the epigenetic regulator SIRT6 in cancer metabolism and how SIRT6 can modulate these metabolic genes expression and its possible use as a therapeutic target for cancer treatment.
\end{abstract}

Key Words: cancer, Warburg effect, SIRT6, glycolysis

ancer is the second leading cause of death in USA and affects $\mathcal{C}$ one in three people during their lifetime. Cancer exhibits major health burden on the society across the world. According to the National Cancer Institute (NIH), in 2019, roughly 1.76 million new cases are expected to be identified and 0.6 million people are expected to die with cancer in the USA. ${ }^{(1)}$ Although, tumors are heterogenic and possess different characteristics, some basic characteristics remain the same which are called "hallmarks of cancer".(2) These hallmarks include six main characteristics composed of hyper-proliferation, evading growth suppressors, angiogenesis, invasion and metastasis, resistance to cell death, and replicative immortality. In addition to these six capabilities, two emerging hallmarks have been added resistance to immune destruction and cell metabolism reprogramming. ${ }^{(3)}$ Cancer cells reprogram its glucose metabolism to aerobic glycolysis with diminished oxidative phosphorylation. ${ }^{(2,4)}$ This reprogramming of glucose metabolism is implicated in resistance to many radiation and chemotherapy wherein numerous physiological activities get altered including DNA repair mechanism, induction of autophagy, and alterations in tumor microenvironment. However, additional evidence is needed to demonstrate the molecular mechanisms linking radiation/chemotherapy resistance to cellular metabolism. ${ }^{(5)}$

Glucose is a key energy source for almost all living cells. Glucose molecules are transported into the cells via glucose transporters (GLUTs). Under sufficient oxygen levels, normal differentiated cells catabolize glucose to $\mathrm{CO}_{2}$ and water, via glycolysis followed by mitochondrial oxidative phosphorylation. This process generates up to 36 ATP per glucose molecule. Under hypoxic conditions, normal differentiated cells ferment glucose to lactate, and this results in the net yield of 2 ATP per glucose molecule (Fig. 1)..$^{(4,6-8)}$ Unlike normal cells, cancerous cells uptake higher amount of glucose and they catabolize most of it to lactic acid regardless of oxygen availability, a phenomenon titled as "Warburg effect" or "Aerobic glycolysis" (Fig. 1). ${ }^{(4,6-8)}$

Looking at the number of generated ATP, one might wonder why cancer cells use more glucose and metabolize it in a less efficient way by producing $\sim 18$-fold less ATP. There are some proposals for this dilemma. First, because non-oxidative phosphorylation fewer metabolic steps process, tumor cells can produce ATP at 10-100 times faster rate than through long oxidative metabolic process. ${ }^{(9)}$ Second, proliferating cells need biomacromolecules (nucleic acids, fatty acids, and amino acids) to generate daughter cells in mitosis, hence, fermentation can provide these building blocks to rapidly generate the needed biomacromolecules. ${ }^{(4,8)}$ Third, due to the accumulation of glycolytic intermediates, high NADPH is derived from the enhanced pentose phosphate pathway (PPP). This enables cancer cells to have sufficient levels of reduced forms of glutathione thus rendering resistance to chemotherapeutic agents. ${ }^{(10)}$ Fourth, tumor cells can survive the hypoxic conditions if oxygen becomes a limitation. ${ }^{(11)}$ Finally, lactic acid is toxic to immune cells and this in turn helps the cancer cells to escape the immune surveillance and promotes angiogenesis. ${ }^{(8)}$

Warburg effect inhibition is one of the proposed therapeutic strategies to control cancer. However, the major limitation of this therapeutic approach is that it will harm the normal cells that use glycolysis for generating energy. Therefore, understanding how to inhibit glycolysis locally and specifically in cancer cells without affecting the normal cells can lead us to find a more suited therapy. ${ }^{(7)}$ Cancer cells have numerous properties including high proliferation, invasion, metastasis, and survival. ${ }^{(3)}$ It is possible that Warburg effect can enhance these properties which are usually associated with oncogenic mutations or signaling pathways. ${ }^{(7)}$ For example, cancer metastasis put the cells in a high oxidative stress. The metabolic tuning to Warburg reduces this oxidative stress by constraining the mitochondrial oxidative metabolism, thereby enhancing cell metastasis. ${ }^{(12)}$ Further, some gene transcriptions are dysregulated to gear the cell metabolism toward aerobic glycolysis. ${ }^{(13)}$

Warburg effect is regulated through the coding genes of key glycolysis enzymes including glucose transporter 1 (GLUT1), hexokinase 2 (HK2), glyceraldehyde 3-phosphate dehydrogenase (GAPDH), phosphoglycerate kinase (PGK1), enolase 1 (ENO1),

To whom correspondence should be addressed. E-mail: nzalazzam@just.edu.jo 
Normal differentiated cells

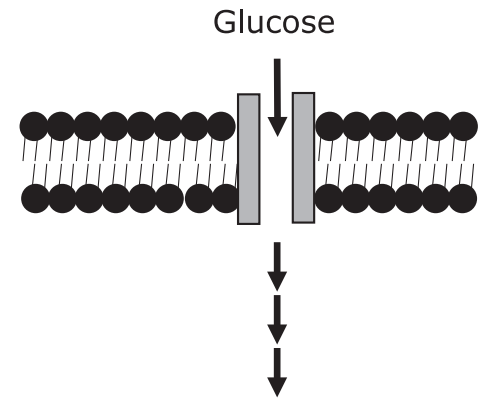

Pyruvate

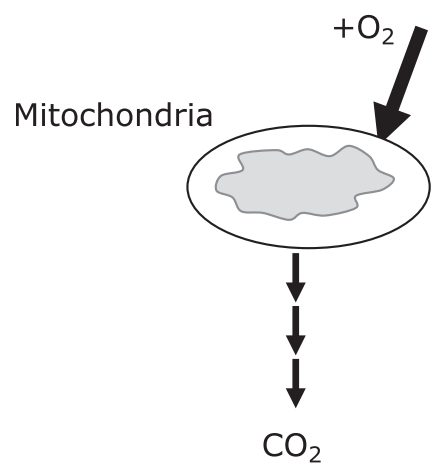

36 ATP

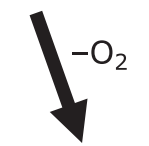

Lactic acid

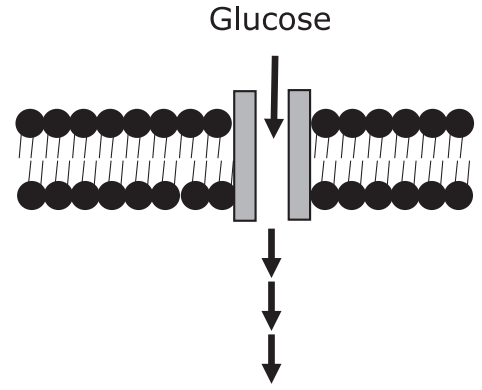

Pyruvate

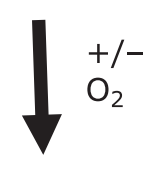

Lactic acid

Fig. 1. A schematic diagram explains glucose metabolism of normal differentiated cells vs cancer cells. Normal differentiated cells can either catabolize glucose to $\mathrm{CO}_{2}$ and water via oxidative phosphorylation under oxygen sufficient conditions or to lactic acid mainly under hypoxic conditions. This generates up to 36 ATP vs 2 ATP molecules per glucose molecule respectively. However, cancerous cells catabolize glucose to lactic acid regardless of oxygen availability, a phenomenon titled as "Warburg effect" or "Aerobic glycolysis". Multiple consecutive arrows indicate different metabolic steps and thick arrows depict the main metabolic pathway used in the cell.

pyruvate kinase-M2 splice isoform (PKM2), and lactate dehydrogenase-A (LDH-A). In addition, Warburg effect is also regulated by transcription factors that regulate the transcription of these glycolytic genes, such as hypoxia-inducible factor-1 alpha $(\mathrm{HIF}-1 \alpha)$ and c-MYC, and by the epigenetic regulator sirtuin 6 (SIRT6). ${ }^{(14)}$ Warburg effect upregulates glucose transporter GLUT1 and downregulates mitochondrial respiration to convert glucose to lactate. $^{(15)}$ After entering the cell, glucose is phosphorylated to glucose 6-phosphate by enzyme hexokinase (HK). HK levels are also elevated in cancer cells. ${ }^{(16)}$ Then glucose 6-phosphate is converted to phosphoenolpyruvate (PEP) via a series of metabolic enzymes. PEP along with one ADP molecule are converted to pyruvate and ATP respectively via pyruvate kinase (PK). ${ }^{(17)}$ Although different PK isoforms are expressed in the human body, PKM2 is the most common isoform expressed in cancer tissues and it is linked to aerobic glycolysis. ${ }^{(18)}$ Under normoxic conditions, pyruvate, the final product of glycolysis, is transported into the mitochondria to be metabolized to carbon dioxide and water via series of reactions. Under hypoxic conditions, pyruvate is interconverted directly to lactate via LDH enzyme. ${ }^{(19)}$ Another player in Warburg effect is the epigenetic factor SIRT6, which has been identified to play a role in metabolic control as well as the tumor suppression. Loss of SIRT6 can lead to increased aerobic glycolysis and enhanced tumorigenesis. ${ }^{(20)}$

SIRT6 is a histone $\mathrm{H} 3$ deacetylates that can bind to the telomere and modulate its function. ${ }^{(21,22)}$ SIRT6 plays a role in extending mammalian lifespan and suppressing various age-related patho- logies, including cancer and metabolic diseases. ${ }^{(22,23)}$ An in vivo study has shown that loss of SIRT6 caused increased glucose fermentation and tumor initiation and growth. The inhibition of glycolysis rescued the tumorigenesis in SIRT6-deficient cells, proving that SIRT6 loss-mediated tumorigenesis is intermediated through its glycolysis upregulation. ${ }^{(24)}$ SIRT6 controls the expression of multiple glycolytic genes. SIRT6 deficient cells have an increased activity of HIF-1 $\alpha$, an increased uptake of glucose, an up-regulation of the main glycolytic genes including GLUT1, PFK1, and LDH, and a downregulation of mitochondrial respiration. ${ }^{(25)}$ In this review, we will mainly highlight the role of the glucose transporter GLUT1, HK2, PKM2, LDH-A, in Warburg effect and cancerogenesis and how these genes expression is modulated by SIRT6. Many studies that link SIRT6 to the mentioned glycolytic genes will be summarized.

\section{GLUT1}

Glucose transmembrane transport is the first step of glucose metabolism and a rate limiting step of glycolysis. ${ }^{(26)}$ GLUT proteins, encoded by $S L C 2 A$ gene, are a family of transporters that facilitate glucose transportation into the cells. According to sequence similarity and function, GLUTs are divided into three classes named as Class I (GLUT 1-4), Class II (GLUT 5, 7, 9, and 11) and Class III (GLUT 6, 8, 10, and 12), and the protonmyoinositol symporter (HMIT1). ${ }^{(27)}$ Of class I transporters, GLUT1 is mainly expressed in erythrocytes, GLUT2 is mainly found in the 
liver, GLUT3 is found in brain, and GLUT4 is found in muscular/ adipose tissue. ${ }^{(28)}$ Among them, GLUT1 is the most important and commonly expressed isoform in various human types of carcinoma. ${ }^{(26,29,30)}$

Using glucose fermentation, cancerous cells practice a less efficient form of metabolism in terms of ATP production, however, they compensate that by increasing glucose uptake to get the needed energy for proliferation. Elevated GLUT1 expression is observed in mucoepidermoid carcinoma cells, which is the most common salivary gland tumor. ${ }^{(31)}$ Apart from mucoepidermoid carcinoma, breast cancer, ${ }^{(32)}$ cervical cancer, ${ }^{(33)}$ prostate cancer, ${ }^{(26)}$ renal carcinoma, ${ }^{(34)}$ and many other cancers also have higher expression of GLUT1 facilitative transporters. ${ }^{(32)}$ Furthermore, Oh et al. ${ }^{(32)}$ showed that GLUT1 does not only act as a glucose transporter, but also plays a role as a regulator of signaling pathways in the carcinogenesis of breast cancer. Knockdown of GLUT1 in breast cancer cells, decreases cell proliferation, deprives the cells of glucose, and reduces cell migration and invasion through attenuation of the EGFR/MAPK signaling pathway as well as the integrin $\beta 1 / \mathrm{Src} / \mathrm{FAK}$ signaling pathways. ${ }^{(32)}$ In addition, GLUT1 mRNA is increased in the peripheral blood of stage II and III colorectal cancer patients compared to stage I patients, suggesting that GLUT1 has a potential to be employed as a molecular marker of hypoxia experienced by tumors circulating in the blood of cancer patients. ${ }^{(29)}$ A meta-analysis that explored the association between GLUT1 and survival in human cancers identified that increased expression of GLUT1 is linked to unfavorable overall survival, poorer disease-free survival, poorer differentiated tumors, positive lymph node metastasis, and larger tumor size. This indicates that targeting GLUT1 can be a good therapeutic approach for various types of cancers. ${ }^{(30)}$

To test if targeting GLUT1 can be a therapeutic option for cancer, an in vitro study showed that blocking GLUT1 transporters either on the genetic level or by using synthetic chemicals attenuated the proliferation and growth of renal cancerous cells via decreasing cellular glucose uptake. ${ }^{(34)}$ Furthermore, an in vivo study showed that knocking down GLUT1 reduces tumor growth in the mouse tumor xenograft model of prostate cancer. ${ }^{(26)}$ Further, the pharmacological or genetic inhibition of GLUT1 in stem cell carcinoma decreased these cells self-renewal potential and deprived their tumor-initiating capacity. ${ }^{(35)}$ Other studies also have shown that attenuating GLUT1 reduces tumorigenicity of tumors including lung carcinoma, ${ }^{(36)}$ ovarian cancer, ${ }^{(37)}$ and head and neck cancer. ${ }^{(38)}$ Moreover, the recently approved drug for diabetes, Canagliflozin, showed a reduction of blood glucose and inhibited complex-I supported respiration and cellular proliferation in prostate and lung cancer cells. ${ }^{(39)}$ All these studies together suggest that GLUT1 is an important player in cancer cell metabolism and targeting it can be a good therapeutic approach especially in cancers that overexpress GLUT1. This can be achieved by reducing the availability of glucose to cancer cells and consequently the available energy for proliferation and growth of tumor cells.

\section{Hexokinase (HK)}

HKs catalyze the first step of glycolytic pathway by irreversibly phosphorylating glucose to glucose 6-phosphate. There are four isoforms of HKs that are encoded by their separate corresponding genes: HK1, HK2, HK3, and HK4 (HK4 is also known as glucokinase). HK 1 is widely expressed in almost all mammalian tissues and highly expressed in the brain, HK2 is expressed in insulinsensitive tissues such as adipose tissue and skeletal muscle, HK3 is expressed at low levels in most tissues, and HK4 expression is limited to the pancreatic B cells and liver. ${ }^{(40)}$ Amongst these, HK2 is the most upregulated isoform in many tumors including glioma, ${ }^{(40-42)}$ prostate cancer, ${ }^{(43)}$ ovarian, ${ }^{(44)}$ hepatocellular, ${ }^{(45)}$ and head and neck cancers. ${ }^{(46)}$

The mitochondrial receptor for HK2, the voltage dependent anion channel (VDAC), facilitates the binding of HK2 to the mitochondria, forming HK2-VDAC complex. ${ }^{(1)}$ The HK2-VDAC complex enhances glycolysis via suppressing the negative feedback of glucose 6-phosphate and enhances ATP production to promote aerobic glycolysis in the presence of oxygen. ${ }^{(41)}$ Furthermore, role of HK2 is also observed at the mRNA/posttranscriptional level in prostate cancer. Clinical and experimental findings have implicated that genetic loss of both Pten and p53 in prostate epithelial cells play a causal role in prostate cancer. This genetic loss mediates tumorigenic pathogenesis by upregulating HK2 at the mRNA/posttranscriptional level by the p53-miR143 axis and at the protein/translational level by PTEN-AKT-mTOR-4EBP1 signaling axis. ${ }^{(43)}$

In contrast to the tumors that express both HK1 and HK2 isoforms, knocking HK2 isoform in cancers that express only HK2 and not HK1 result in the inhibition of tumor cell proliferation, colony formation, and xenograft tumor progression. ${ }^{(47)}$ Contradictorily, another study used HK2 conditional knockout mice and showed that HK2 plays a crucial role in tumor initiation and maintenance in mouse models of KRas-driven lung cancer, and ErbB2-driven breast cancer, regardless of HK1 expression. Further, systemic deletion of HK2 reduces the burden in mice bearing lung tumors without adverse physiological consequences. ${ }^{(48)}$

\section{Pyruvate Kinase (PK)}

Microarray studies in cancer have shown a dysregulation in many glycolytic genes. One of these genes is PK, which encodes the enzyme that generates pyruvate from PEP as a final step of glycolysis. ${ }^{(7,18)} \mathrm{PK}$ has four isoforms; $\mathrm{R}$ found in the red blood cells, $\mathrm{L}$ in the liver, $\mathrm{M} 1$ in most adult tissues, and M2 in embryonic tissues and usually detected in tumors. ${ }^{(49)}$ The M2 isoform exists in dimeric and tetrameric forms and the ratio between those two isoforms in cancer cells is influenced by different oncogenic proteins. At physiological conditions, the dimeric form has less affinity towards its substrate PEP and acts as an active nuclear protein kinase whereas the tetrameric form has a high affinity toward PEP. ${ }^{(2)}$

It is well known now that the dominant structure of PKM2 in cancer is dimeric. The dimeric form help in the accumulation of the phosphometabolites upstream of pyruvate kinase, such as PEP, glycerate 3-P, glyceraldehyde 3-P, fructose 1,6-P2, ribose-5P, and 5-ribose-PP, thereby increases the availability of biomass precursors (nucleic acid, amino acid and phospholipid) ${ }^{(50)}$ Further, the nuclear dimeric form of PKM2 acts as a kinase that directly phosphorylates STAT3 at Y705 position thereby activating the transcription of a number of STAT3 targeted genes and histone $\mathrm{H} 3$ at T11. ${ }^{(51,52)}$ PKM2 dimer also promotes cell proliferation and tumor growth, explaining the important role of the dimeric PKM2 in cancer progression. ${ }^{(51,52)}$ Moreover, a subcellular compartmentalization of PKM2 may characterize therapeutic response of non-small cell lung cancer (NSCLC) tumor sections of responding and nonresponding mice to anti-PKM2 therapy. It was demonstrated that mice with reduced cytoplasmic PKM2 with low or undetectable nuclear compartmentalization had a good response to anti-PKM2 therapy. In contrast, mice with increased PKM2 nuclear staining and low or undetectable cytoplasmic staining were non-responding. ${ }^{(53)}$

In vitro and in vivo studies show that a switch from PKM1 to PKM2 isoform enhances cell proliferation and consequently increases tumor size and mass. ${ }^{(18)}$ Increased PKM2 expression can not only increase lactate production, but it also induce reductive glutamine metabolism. Reductive glutamine metabolism enhances cell proliferation under hypoxic conditions, which facilitates tumor growth. ${ }^{(54)}$ PKM2 deletion reduces tumorigenesis, increases the survival rate, ${ }^{(18)}$ and reverses Warburg effect in tumor associated stromal cells. ${ }^{(55)}$ Moreover, PKM2 mediates cancer metastasis in many tumors including gastric and hepatic cancers. ${ }^{(55,56)}$ PKM2 
induces angiogenesis through STAT3 downstream signaling and upregulates HIF- $1 \alpha$ via NF- $\kappa \mathrm{B}$ pathway. ${ }^{(57,58)}$ In addition, cantharidin - an anticancer active compound - inhibits metastasis in breast cancer mainly through its downregulation of GLUT1 and PKM2. ${ }^{(55)}$

In contrast, other study shows that PKM2 loss promotes mammary tumor formation in a Brca1-loss-driven model of breast cancer and activation of PKM2 inhibits tumor growth. ${ }^{(59)}$ Also, the germline absence of PKM2 potentiates metabolic distress in hepatic carcinoma. ${ }^{(15)}$ These contradictory studies of PKM2 inhibition highlight the importance of understanding the contextdependent metabolic needs of each cancer to effectively target its metabolism for therapeutic benefit.

\section{Lactate Dehydrogenase (LDH)}

Lactate dehydrogenase is composed of four subunits (tetramer). The two most common monomers are the LDH-M and LDH-H. These two subunits can form five possible tetramers: H4, H3M1, $\mathrm{H} 2 \mathrm{M} 2$, H1M3, and M4. The modern terminology designates the monomers as A (LDH-M) and B (LDH-H). ${ }^{(60)}$ LDH-A levels are increased in many tumors and it can be used as predictor for tumor malignancy, recurrence, survival and metastasis. ${ }^{(61)}$ LDH-A high level promotes a metabolic rewiring to aerobic glycolysis, generating lactic acid as a by-product. ${ }^{(62)}$ Interestingly, the phosphorylation status of LDH-A at tyrosine 10 may also serve as a biomarker for assessing treatment response to human epidermal growth factor receptor 2 (HER2) and avian sarcoma viral oncogene $\mathrm{V}$-src homolog (Src) inhibitors. Tyrosine 10 phosphorylation is commonly upregulated in metastatic tumors compared to primary tumors in human breast cancer, activates LDH-A, and provides a pro-invasive and pro-metastatic potential to cancer cells. ${ }^{(63)}$

LDH-A is overexpressed in urinary bladder cancer cells via PLCE/STAT3 upstream signaling access. ${ }^{(64)}$ Overexpression of LDH-A promotes cell proliferation by enhancing glycolysis in urinary bladder carcinoma and hepatocellular carcinoma. ${ }^{(64,65)}$ In addition, the elevated level of LDH-A is highly correlated with poor degree of histological differentiation, as well as the disease stage in hepatocellular carcinoma and pancreatic carcinoma patients. ${ }^{(65,66)}$ High levels of LDH-A protein in human esophageal squamous cell carcinoma tissues correlate with tumor metastasis, stage, and survival. ${ }^{(67)}$ Furthermore, high serum levels of LDH is linked to the radiation and chemotherapy resistance in sarcomas, lymphomas, and carcinomas. ${ }^{(68)}$

Targeting LDH-A can be beneficial for cancer therapy. The $L D H-A$ deletion in the triple-transgenic mouse model of prostate cancer significantly reduced lactate to pyruvate ratio, causing tumor reduction. This data suggests that $\mathrm{LDH}$ activity and lactate are required for tumor progression. ${ }^{(69)}$ Also, in another study, inhibiting LDH-A using siRNAs or small molecule inhibitors reduced cell proliferation, migration, and invasion; and increased cell apoptosis, and downregulated proteases involved in extracellular matrix degradation and tumor metastasis of prostate cancer. ${ }^{(70)}$

\section{SIRT6 and Its Effect on Glycolytic Players}

Sirtuins are $\mathrm{NAD}^{+}$-dependent deacetylases that are involved in metabolism, aging and inflammation. Mammals have seven types of sirtuins (SIRT 1-7). Amongst these, SIRT6 seems to have a special role in regulating metabolism and lifespan. ${ }^{(20,71)}$ SIRT6 is a telomere binding factor that deacetylates histone H3 lysine 9 (H3K9) and lysine 56 (H3K56). ${ }^{(21,22)}$ Upon histone 3 deacetylation, SIRT6 attenuates the transcriptional activity of many transcription factors including NF- $\mathrm{\kappa B}$, HIF 1, c-MYC, and JUN to inhibit target gene expression. ${ }^{(8)}$ SIRT6 plays a role in extending mammalian lifespan and suppressing various age-related pathologies, including cancer and metabolic diseases. ${ }^{(22,23)}$ SIRT6 KO mice are born without abnormalities, however around 2-3 weeks of age they rapidly start to develop a severe deteriorating phenotype like reduced lymphocytes number, subcutaneous fat loss, and severe hypoglycemia that leads to their death around the 4 th week of age. ${ }^{(72)}$ A study has shown that loss of SIRT6 in animals caused increased glucose fermentation and tumor initiation and growth. The inhibition of glycolysis rescued the tumorigenesis in SIRT6-deficient cells, proving that SIRT6 loss-mediated tumorigenesis is intermediated through its glycolysis upregulation. ${ }^{(24)}$ Notably, a recent study demonstrated that SIRT6 overexpression can reinstate glucose homeostasis and inhibit lipo-toxicity in high fat diet-induced obesity. ${ }^{(73)}$ This further improves SIRT6 role in glucose homeostasis.

SIRT6 controls the expression of multiple glycolytic genes, it acts as a corepressor of the transcription factor HIF-1 $\alpha$, a main regulator of nutrient stress responses. SIRT6 deficient cells have an increased activity of HIF-1 $\alpha$, an increased uptake of glucose, an up-regulation of the main glycolytic genes including GLUT1, PFK1, and LDH, and a downregulation of mitochondrial respiration. ${ }^{(25)}$ HIF- $1 \alpha$ is a main regulator of cancer progression and metastasis; it regulates transcription of many genes including the genes involved in glucose metabolism, tumor invasion, and tumor metastasis. ${ }^{(74)}$ A separate study showed that SIRT6 overexpression inhibited HIF-1 $\alpha$ expression and tumor angiogenesis in lung cancer. ${ }^{(75)}$ In another study carried on breast cancer cells, the transcription factor RUNX2-mediated suppression of SIRT6 led to increased glucose uptake, upregulated GLUT1 and HK2 levels, increased glycolysis, activated pyruvate dehydrogenase kinase to inhibit pyruvate dehydrogenase, and downregulated mitochondrial respiration in the tumor microenvironment. ${ }^{(76)}$ SIRT6 is known to be a negative regulator for HK2. ${ }^{(77,78)}$ Furthermore, the tumor suppressor FKHRL1 (also named as FOXO3a) is shown to promote SIRT6 transcription, thus inhibiting Warburg effect in glioblastoma by attenuating the expression of a cluster of glycolytic genes including GLUT1, HK2, and LDH-A. ${ }^{(79)}$ FOXO3a enhances SIRT6 expression not only in glioblastoma tumor but also in colon cancer cells. ${ }^{(79,80)}$ The enhanced SIRT expression inhibits the expression of downstream survival and metabolic genes by deacetylating the histone at $\mathrm{H} 3 \mathrm{~K} 9$ site in the promoter region. ${ }^{(79,80)}$ Furthermore, SIRT6 is also downregulated in other cancers including hepatocellular carcinoma, ${ }^{(81)}$ colorectal cancer, ${ }^{(82)}$ and ovarian cancer. ${ }^{(83)}$

In addition, SIRT6 deacetylates the nuclear PKM2 at K433 residue resulting in the nuclear export of PKM2. As a result, SIRT6 suppresses the oncogenic functions of PKM2, turning out in reduced cell proliferation, migration, and invasion. Moreover, reduced SIRT6 levels is linked to increased nuclear acetylated PKM2 levels in higher tumor grades of hepatocellular carcinoma. ${ }^{(84)}$ Acetylation of PKM2 does not prevent enzyme activation however it promotes the nuclear accumulation and protein kinase activity of PKM2. ${ }^{(85)}$ SIRT6 expression is reduced in nasal polyp fibroblasts, resulting in upregulation of LDH enzyme and consequently enhanced lactate production. ${ }^{(86)}$ In a study performed on hepatocellular carcinoma (HCC), SIRT6 deacetylated the heterogeneous nuclear ribonucleoproteins (hnRNP) A1 at certain lysine residues. The deacetylated hnRNP Al inhibited glycolysis, cell proliferation, and the alternative splicing of PKM mRNA to PKM2 isoform in HCC cells. The same study also presented the immunohistochemistry of $\mathrm{HCC}$ with an increase in the acetylated hnRNP in the tumor tissue compared with the adjacent tissue. ${ }^{\left({ }^{87}\right)}$ This might suggest a decreased level of SIRT6 and its deacetylation activity in these tumor tissues.

Despite all the previously discussed data supporting SIRT6 role as a tumor suppressor, few studies have shown that SIRT6 could function as an oncogene in some cancers. Yang et al. ${ }^{\left({ }^{88}\right)}$ proved that SIRT6 induces tumorigenesis and Warburg effect in papillary thyroid cancer by upregulating reactive oxygen species. However, SIRT6 acts as an antioxidant in mesenchymal stem cells by activating NRF2. ${ }^{(89)}$ SIRT6 downregulation in adrenocortical 
carcinoma increases tumorigenesis and ROS via activation of NF- $\kappa \mathrm{B}$ signaling. ${ }^{(90)}$ Others have also shown a positive correlation of SIRT6 and acute myeloid leukemia (AML), and chronic lymphocytic leukemia (CLL). ${ }^{(91)}$

\section{Conclusion and Future Direction}

Cancer cells use glucose metabolism reprogramming to ferment glucose molecules to lactate instead of mitochondrial metabolism, this is known as "Warburg effect". Overall, most studies have indicated a positive role for SIRT6 in tumor suppression. This anti-tumor activity is mediated through different mechanisms such as controlling ROS levels, attenuating the expression of downstream survival protein, and rewiring of cell metabolism. SIRT6 deacetylate the promotor of HIF-1 $\alpha$, a main transcription regulator of glycolytic genes, causing its corepression. Loss of SIRT6 in cancers modulate the metabolism via different pathways (Fig. 2). It increases HIF-1 $\alpha$ and thereby increases the expression of many glycolytic genes including GLUT1, and LDH-A. Also, SIRT6 can reduce PKM2 splicing via deacetylation of hnRNP, or it can directly deacetylate PKM2 at k433 which enhances its nuclear transport and reduces its oncogenic function. SIRT6 also enhances the expression of HK2, however, which transcription factor is mediating this role is not yet known. Upon upregulation of glycolytic genes, the cells will rewire its metabolism to aerobic glycolysis which promote cell proliferation, invasion, and metastasis.

Although these studies provide a convincing evidence for a tumorigenic role of SIRT6 in metabolic reprogramming, more research is needed to know the exact signaling cascade of SIRT6 mediated metabolic rewiring. Further, finding a good SIRT6 activator as a therapeutic option for cancer and studying which molecular features of cancer will be attenuated by enhancing SIRT6 expression/activity needs to be determined.

\section{Acknowledgments}

The author acknowledges the medical writer "Prachi Patil" for her great assistance in editing the manuscript and Jordan University of Science and Technology for the Support.

\section{Abbreviations}

$\begin{array}{ll}\text { GLUT } & \text { glucose transporter } \\ \text { HIF } & \text { hypoxia inducible factor } \\ \text { HK } & \text { hexokinase } \\ \text { hnRNP } & \text { heterogeneous nuclear RiboNucleoProteins } \\ \text { LDH } & \text { lactate dehydrogenase } \\ \text { PEP } & \text { phosphoenolpyruvate } \\ \text { PK } & \text { pyruvate kinase } \\ \text { PKM2 } & \text { pyruvate kinase-M2 splice isoform } \\ \text { SIRT6 } & \text { sirtuin 6 }\end{array}$

\section{References}

1 Siegel RL, Miller KD, Jemal A. Cancer statistics, 2019. CA Cancer J Clin 2019; 69: 7-34.

2 Rihan M, Nalla LV, Dharavath A, Shard A, Kalia K, Khairnar A. Pyruvate kinase M2: a metabolic bug in re-wiring the tumor microenvironment. Cancer Microenviron 2019; 12: 149-167.

3 Hanahan D, Weinberg RA. Hallmarks of cancer: the next generation. Cell 2011; 144: 646-674.

4 Vander Heiden MG, Cantley LC, Thompson CB. Understanding the Warburg effect: the metabolic requirements of cell proliferation. Science 2009; 324: 1029-1033.

5 Lin J, Xia L, Liang J, et al. The roles of glucose metabolic reprogramming in chemo- and radio-resistance. J Exp Clin Cancer Res 2019; 38: 218.

6 Voet D, Voet J, Pratt C. Fundamentals of Biochemistry: Life at the Mole-

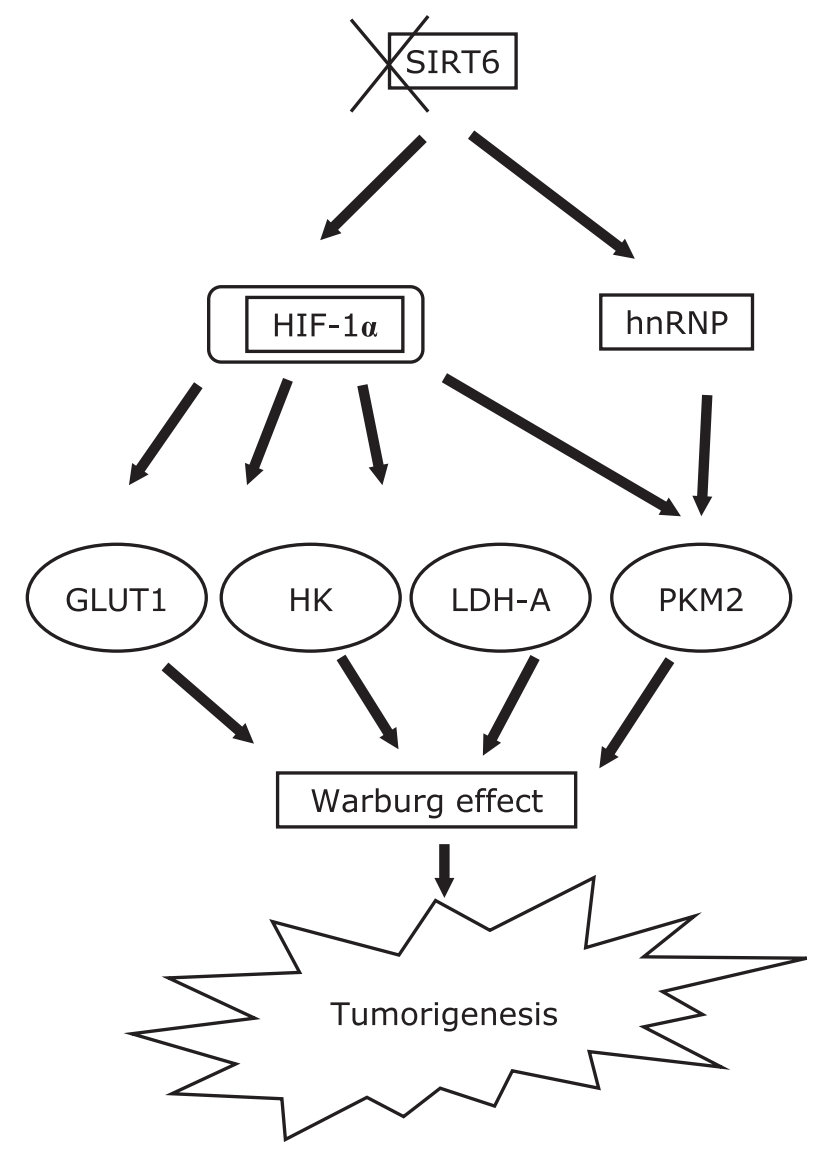

Fig. 2. A schematic diagram explains the effect of SIRT6 loss on Warburg effect. Loss or downregulation (indicated by $X$ symbol) of SIRT6 and its mediated deacetylation in many cancers lead to increased expression of the glycolytic genes GLUT1, HK2, PKM2, and LDH-A and consequently induces Warburg effect and tumorigenesis. In part this is mediated by activation of HIF- $1 \alpha$ that in turn mediates glycolytic gene expression and the acetylated hnRNP that enhances PKM2 splicing.

\section{Conflict of Interest}

No potential conflicts of interest were disclosed.

\section{Compliance with Ethical Standards}

This article does not contain descriptions of studies performed by the author with participation of humans or using animals as objects. 
the "Warburg effect", i.e., elevated glycolysis in the presence of oxygen. $J$ Bioenerg Biomembr 2007; 39: 211-222.

$12 \mathrm{Lu} \mathrm{J}$. The Warburg metabolism fuels tumor metastasis. Cancer Metastasis Rev 2019; 38: 157-164.

13 Li L, Liang Y, Kang L, et al. Transcriptional regulation of the Warburg effect in cancer by SIX1. Cancer Cell 2018; 33: 368-385.

14 Yu W, Yang Z, Huang R, Min Z, Ye M. SIRT6 promotes the Warburg effect of papillary thyroid cancer cell BCPAP through reactive oxygen species. Onco Targets Ther 2019; 12: 2861-2868.

15 Dayton TL, Gocheva V, Miller KM, et al. Germline loss of PKM2 promotes metabolic distress and hepatocellular carcinoma. Genes Dev 2016; 30: 1020 1033.

16 Sun L, Shukair S, Naik TJ, Moazed F, Ardehali H. Glucose phosphorylation and mitochondrial binding are required for the protective effects of hexokinases I and II. Mol Cell Biol 2008; 28: 1007-1017.

17 Israelsen WJ, Vander Heiden MG. Pyruvate kinase: function, regulation and role in cancer. Semin Cell Dev Biol 2015; 43: 43.

18 Christofk HR, Vander Heiden MG, Harris MH, et al. The M2 splice isoform of pyruvate kinase is important for cancer metabolism and tumour growth. Nature 2008; 452: 230-233.

19 Gray LR, Tompkins SC, Taylor EB. Regulation of pyruvate metabolism and human disease. Cell Mol Life Sci 2014; 71: 2577-2604.

20 Zhong L, Mostoslavsky R. SIRT6. Transcription 2010; 1: 17-21.

21 Polyakova O, Borman S, Grimley R, Vamathevan J, Hayes B, Solari R. Identification of novel interacting partners of Sirtuin6. PLoS One 2012; 7: e51555.

22 Giblin W, Skinner ME, Lombard DB. Sirtuins: guardians of mammalian healthspan. Trends Genet 2014; 30: 271-286.

23 Kitada M, Ogura Y, Monno I, Koya D. Sirtuins and type 2 diabetes: role in inflammation, oxidative stress, and mitochondrial function. Front Endocrinol (Lausanne) 2019; 10: 187.

24 Sebastián C, Zwaans BM, Silberman DM, et al. The histone deacetylase SIRT6 is a tumor suppressor that controls cancer metabolism. Cell 2012; 151: 1185-1199.

25 Zhong L, D’Urso A, Toiber D, et al. The histone deacetylase Sirt6 regulates glucose homeostasis via Hif1 $\alpha$. Cell 2010; 140: 280-293.

26 Xiao H, Wang J, Yan W, et al. GLUT1 regulates cell glycolysis and proliferation in prostate cancer. Prostate 2018; 78: 86-94.

27 Joost HG, Bell GI, Best JD, et al. Nomenclature of the GLUT/SLC2A family of sugar/polyol transport facilitators. Am J Physiol Endocrinol Metab 2002; 282: E974-E976.

28 Yamamoto T, Seino Y, Fukumoto H, et al. Over-expression of facilitative glucose transporter genes in human cancer. Biochem Biophys Res Commun 1990; 170: 223-230.

29 Chung FY, Huang MY, Yeh CS, et al. GLUT1 gene is a potential hypoxic marker in colorectal cancer patients. BMC Cancer 2009; 9: 241.

30 Yu M, Yongzhi H, Chen S, et al. The prognostic value of GLUT1 in cancers: a systematic review and meta-analysis. Oncotarget 2017; 8: 43356-43367.

31 Demasi APD, Costa AF, Altemani A, Furuse C, Araújo NS, Araújo VC. Glucose transporter protein 1 expression in mucoepidermoid carcinoma of salivary gland: correlation with grade of malignancy. Int J Exp Pathol 2010; 91: $107-113$.

32 Oh S, Kim H, Nam K, Shin I. Glut1 promotes cell proliferation, migration and invasion by regulating epidermal growth factor receptor and integrin signaling in triple-negative breast cancer cells. BMB Rep 2017; 50: 132-137.

33 Rudlowski C, Becker AJ, Schroder W, Rath W, Büttner R, Moser M. GLUT1 messenger RNA and protein induction relates to the malignant transformation of cervical cancer. Am J Clin Pathol 2003; 120: 691-698.

34 Chan DA, Sutphin PD, Nguyen P, et al. Targeting GLUT1 and the Warburg effect in renal cell carcinoma by chemical synthetic lethality. Sci Transl Med 2011; 3: 94ra70.

35 Shibuya K, Okada M, Suzuki S, et al. Targeting the facilitative glucose transporter GLUT1 inhibits the self-renewal and tumor-initiating capacity of cancer stem cells. Oncotarget 2014; 6: 651-661.

36 Liao H, Wang Z, Deng Z, Ren H, Li X. Curcumin inhibits lung cancer invasion and metastasis by attenuating GLUT1/MT1-MMP/MMP2 pathway. Int J Clin Exp Med 2015; 8: 8948-8957.

37 Gwak H, Haegeman G, Tsang BK, Song YS. Cancer-specific interruption of glucose metabolism by resveratrol is mediated through inhibition of Akt/ GLUT1 axis in ovarian cancer cells. Mol Carcinog 2015; 54: 1529-1540.

38 Wang YD, Li SJ, Liao JX. Inhibition of glucose transporter 1 (GLUT1) chemosensitized head and neck cancer cells to cisplatin. Technol Cancer Res Treat 2013; 12: 525-535.

39 Villani LA, Smith BK, Marcinko K, et al. The diabetes medication Canagliflozin reduces cancer cell proliferation by inhibiting mitochondrial complex-I supported respiration. Mol Metab 2016; 5: 1048-1056.

40 Wilson JE. Isozymes of mammalian hexokinase: structure, subcellular localization and metabolic function. $J$ Exp Biol 2003; 206 (Pt 12): 2049-2057.

41 Liu H, Yang H, Wang X, Tu Y. The contribution of hexokinase 2 in glioma. Cancer Transl Med 2018; 4: 54.

42 Wolf A, Agnihotri S, Micallef J, et al. Hexokinase 2 is a key mediator of aerobic glycolysis and promotes tumor growth in human glioblastoma multiforme. J Exp Med 2011; 208: 313-326.

43 Wang L, Xiong $\mathrm{H}, \mathrm{Wu}$ F, et al. Hexokinase 2-mediated Warburg effect is required for PTEN- and p53-deficiency-driven prostate cancer growth. Cell Rep 2014; 8: 1461-1474.

44 Siu MKY, Jiang YX, Wang JJ, et al. Hexokinase 2 regulates ovarian cancer cell migration, invasion and stemness via FAK/ERK1/2/MMP9/NANOG/ SOX9 signaling cascades. Cancers 2019; 11. pii: E813.

45 Yoo JJ, Yu S, Na J, et al. Hexokinase-II inhibition synergistically augments the anti-tumor efficacy of sorafenib in hepatocellular carcinoma. Int J Mol Sci 2019; 20. pii: E1292.

46 Yang H, Zhong JT, Zhou SH, Han HM. Roles of GLUT-1 and HK-II expression in the biological behavior of head and neck cancer. Oncotarget 2019; 10: 3066-3083.

$47 \mathrm{Xu} \mathrm{S}$, Catapang A, Doh HM, et al. Hexokinase 2 is targetable for HK1negative, HK2-positive tumors from a wide variety of tissues of origin. $\mathrm{J} \mathrm{Nucl}$ Med 2019; 60: 212-217.

48 Patra KC, Wang Q, Bhaskar PT, et al. Hexokinase 2 is required for tumor initiation and maintenance and its systemic deletion is therapeutic in mouse models of cancer. Cancer Cell 2013; 24: 213-228.

49 Amin S, Yang P, Li Z. Pyruvate kinase M2: a multifarious enzyme in noncanonical localization to promote cancer progression. Biochim Biophys Acta Rev Cancer 2019; 1871: 331-341.

50 Mazurek S, Boschek CB, Hugo F, Eigenbrodt E. Pyruvate kinase type M2 and its role in tumor growth and spreading. Semin Cancer Biol 2005; 15: 300 308 .

51 Gao X, Wang H, Yang JJ, Liu X, Liu ZR. Pyruvate kinase M2 regulates gene transcription by acting as a protein kinase. Mol Cell 2012; 45: 598-609.

52 Yang W, Xia Y, Hawke D, et al. PKM2 phosphorylates histone H3 and promotes gene transcription and tumorigenesis. Cell 2012; 150: 685-696.

53 Suzuki A, Puri S, Leland P, et al. Subcellular compartmentalization of PKM2 identifies anti-PKM2 therapy response in vitro and in vivo mouse model of human non-small-cell lung cancer. PLoS One 2019; 14: e0217131.

54 Liu M, Wang Y, Ruan Y, et al. PKM2 promotes reductive glutamine metabolism. Cancer Biol Med 2018; 15: 389-399.

55 Pan Y, Zheng Q, Ni W, et al. Breaking glucose transporter 1/pyruvate kinase M2 glycolytic loop is required for cantharidin inhibition of metastasis in highly metastatic breast cancer. Front Pharmacol 2019; 10: 590.

56 Bi YH, Han WQ, Li RF, et al. Signal transducer and activator of transcription 3 promotes the Warburg effect possibly by inducing pyruvate kinase M2 phosphorylation in liver precancerous lesions. World J Gastroenterol 2019; 25: 1936-1949.

57 Chen D, Wei L, Liu ZR, et al. Pyruvate kinase M2 increases angiogenesis, neurogenesis, and functional recovery mediated by upregulation of STAT3 and focal adhesion kinase activities after ischemic stroke in adult mice. Neurotherapeutics 2018; 15: 770-784.

58 Azoitei N, Becher A, Steinestel K, et al. PKM2 promotes tumor angiogenesis by regulating HIF-1 $\alpha$ through NF- $\kappa \mathrm{B}$ activation. Mol Cancer 2016; 15: 3.

59 Israelsen WJ, Dayton TL, Davidson SM, et al. PKM2 isoform-specific deletion reveals a differential requirement for pyruvate kinase in tumor cells. Cell 2013; 155: 397-409.

60 Gladden LB. Lactate as a key metabolic intermediate in cancer. Ann Transl Med 2019; 7: 210.

61 Walenta S, Mueller-Klieser WF. Lactate: mirror and motor of tumor malignancy. Semin Radiat Oncol 2004; 14: 267-274.

62 Valvona CJ, Fillmore HL, Nunn PB, Pilkington GJ. The regulation and function of lactate dehydrogenase A: therapeutic potential in brain tumor. Brain Pathol 2016; 26: 3-17.

63 Jin L, Chun J, Pan C, et al. Phosphorylation-mediated activation of LDHA promotes cancer cell invasion and tumour metastasis. Oncogene 2017; 36: 3797-3806. 
64 Cheng H, Hao Y, Gao Y, et al. PLCe promotes urinary bladder cancer cells proliferation through STAT3/LDHA pathway-mediated glycolysis. Oncol Rep 2019; 41: 2844-2854.

65 Guo Y, Li X, Sun X, et al. Combined aberrant expression of NDRG2 and LDHA predicts hepatocellular carcinoma prognosis and mediates the antitumor effect of gemcitabine. Int J Biol Sci 2019; 15: 1771-1786.

66 Shi M, Cui J, Du J, et al. A novel KLF4/LDHA signaling pathway regulates aerobic glycolysis in and progression of pancreatic cancer. Clin Cancer Res 2014; 20: 4370-4380.

67 Yao F, Zhao T, Zhong C, Zhu J, Zhao H. LDHA is necessary for the tumorigenicity of esophageal squamous cell carcinoma. Tumor Biol 2013; 34: 2531.

68 Miao P, Sheng S, Sun X, Liu J, Huang G. Lactate dehydrogenase A in cancer: a promising target for diagnosis and therapy. IUBMB Life 2013; 65: 904-910.

69 Bok R, Lee J, Sriram R, et al. The role of lactate metabolism in prostate cancer progression and metastases revealed by dual-agent hyperpolarized ${ }^{13} \mathrm{C}$ MRSI. Cancers (Basel) 2019; 11. pii: E257.

70 Xian ZY, Liu JM, Chen QK, et al. Inhibition of LDHA suppresses tumor progression in prostate cancer. Tumor Biol 2015; 36: 8093-8100.

71 Michishita E, McCord RA, Berber E, et al. SIRT6 is a histone H3 lysine 9 deacetylase that modulates telomeric chromatin. Nature 2008; 452: 492-496.

72 Mostoslavsky R, Chua KF, Lombard DB, et al. Genomic instability and aging-like phenotype in the absence of mammalian SIRT6. Cell 2006; 124: 315-329.

73 Kanfi Y, Peshti V, Gil R, et al. SIRT6 protects against pathological damage caused by diet-induced obesity. Aging Cell 2010; 9: 162-173.

74 Liao D, Corle C, Seagroves TN, Johnson RS. Hypoxia-inducible factor- $1 \alpha$ is a key regulator of metastasis in a transgenic model of cancer initiation and progression. Cancer Res 2007; 67: 563-572.

75 Wang J, Sheng Z, Cai Y. SIRT 6 overexpression inhibits HIF1 $\alpha$ expression and its impact on tumor angiogenesis in lung cancer. Int J Clin Exp Pathol 2018; 11: 2940-2947.

76 Choe M, Brusgard JL, Chumsri S, et al. The RUNX2 transcription factor negatively regulates SIRT6 expression to alter glucose metabolism in breast cancer cells. J Cell Biochem 2015; 116: 2210-2226.

77 Gupta P, Sheikh T, Sen E. SIRT6 regulated nucleosomal occupancy affects Hexokinase 2 expression. Exp Cell Res 2017; 357: 98-106.

78 Singh A, Sen E. Reciprocal role of SIRT6 and Hexokinase 2 in the regulation of autophagy driven monocyte differentiation. Exp Cell Res 2017; 360: 365-374.

79 Dong Z, Zhong X, Lei Q, Chen F, Cui H. Transcriptional activation of SIRT6 via FKHRL1/FOXO3a inhibits the Warburg effect in glioblastoma cells. Cell Signal 2019; 60: 100-113.

80 Zhang Y, Nie L, Xu K, et al. SIRT6, a novel direct transcriptional target of FoxO3a, mediates colon cancer therapy. Theranostics 2019; 9: 2380-2394.

81 Marquardt JU, Fischer K, Baus K, et al. Sirtuin-6-dependent genetic and epigenetic alterations are associated with poor clinical outcome in hepatocellular carcinoma patients. Hepatology 2013; 58: 1054-1064.

82 Qi J, Cui C, Deng Q, et al. Downregulated SIRT6 and upregulated NMNAT2 are associated with the presence, depth and stage of colorectal cancer. Oncol Lett 2018; 16: 5829-5837.

83 Zhang J, Yin XJ, Xu CJ, et al. The histone deacetylase SIRT6 inhibits ovarian cancer cell proliferation via down-regulation of Notch 3 expression. Eur Rev Med Pharmacol Sci 2015; 19: 818-824.

84 Bhardwaj A, Das S. SIRT6 deacetylates PKM2 to suppress its nuclear localization and oncogenic functions. Proc Natl Acad Sci U S A 2016; 113: E538-E547.

85 Chen L, Shi Y, Liu S, Cao Y, Wang X, Tao Y. PKM2: the thread linking energy metabolism reprogramming with epigenetics in cancer. Int $\mathrm{J} \mathrm{Mol} \mathrm{Sci}$ 2014; 15: 11435-11445.

86 Shun CT, Lin SK, Hong CY, Lin CF, Liu CM. Sirtuin 6 modulates hypoxiainduced autophagy in nasal polyp fibroblasts via inhibition of glycolysis. $\mathrm{Am}$ J Rhinol Allergy 2016; 30: 179-185.

87 Yang H, Zhu R, Zhao X, et al. Sirtuin-mediated deacetylation of hnRNP A1 suppresses glycolysis and growth in hepatocellular carcinoma. Oncogene 2019; 38: 4915-4931.

88 Yang Z, Yu W, Huang R, Ye M, Min Z. SIRT6/HIF-1 $\alpha$ axis promotes papillary thyroid cancer progression by inducing epithelial-mesenchymal transition. Cancer Cell Int 2019; 19: 17.

89 Pan H, Guan D, Liu X, et al. SIRT6 safeguards human mesenchymal stem cells from oxidative stress by coactivating NRF2. Cell Res 2016; 26: 190 205.

90 Wu X, Tian H, Xue L, Wang L. SIRT6 abrogation promotes adrenocortical carcinoma through activation of NF-кB signaling. Mol Cell Biochem 2019; 458: $1-10$.

91 Khan RI, Nirzhor SSR, Akter R. A review of the recent advances made with SIRT6 and its implications on aging related processes, major human diseases, and possible therapeutic targets. Biomolecules 2018; 8. pii: E44. 